\title{
The Effect of Colors and Positional Noise on Reading Performance with Non-words-Part 2
}

\author{
Abdullah Alsalhi ${ }^{1,2}$, Nadia Northway ${ }^{1}$, Glyn Walsh ${ }^{1}$, Abd Elaziz Mohamed Elmadina $^{2 *}$ \\ ${ }^{1}$ Department of Vision Sciences, Glasgow Caledonian University, Glasgow, UK; ${ }^{2}$ Department of Optometry, Qassim University, \\ Qassim, Saudi Arabia
}

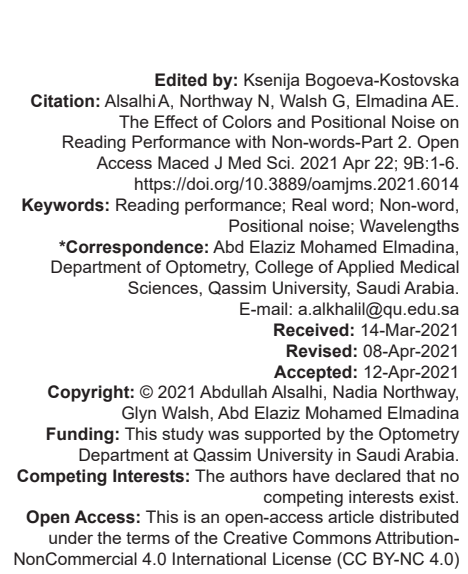

\section{Introduction}

Reading can be described as a complex cognitive process of decryption signs to create meaning. It is also a way of language achievement, communication, and sharing information and ideas. Therefore, reading is a basic requirement for an advanced society. There are three basic processes underlying reading: Sight perception, printed word recognition, and language comprehension [1]. Word recognition is considered a fundamental literacy skill that enables access to and processing of written language, as well as influencing reading performance. Word recognition can be described as the ability to accurately and automatically recognize words, with and without semantic context. It is a stamp of skilled readers' performance or the ability to accurately identify printed words [2]. Positional noise and colors have many effects on reading performance (including the orthographic aspects of reading and word recognition), different effects when non-words are used because reading parallel processing are different for lexical and sublexical items; each of them has functionally independent routes of reading at the wholeword level [1], [2], [3], [4], [5] or $\mathrm{K}$ and $\mathrm{M}$ pathway rapid processing effects. Investigating the reading processes has taken priority in the literature in this field over recent decades. Word recognition can be described as the ability of the reader to identify the written word correctly and virtually effortlessly. This depends on the match between the printed letters and the vocabulary representation. The repaid word recognition is the main factor that makes the reader fluent. This ability can be enhanced in individuals by practicing through speaking and listening [6]. There are two types of coding that affect word recognition - orthographic and graphemic skills and both types of coding are activated in parallel during word recognition [7]. Non-word reading involves some underlying processes, including phonological storage and retrieval, speech motor planning, and execution [3]. According to Joubert and Lecours [2] reported that the non-words and novel words require longer times to be read than regular words [2]. Furthermore, the reading rate for substitutions involving visually similar English letters (non-words), for example, pncblem, problnc, and qroblem, decreases, with reading taking 2.5 times longer than normal. Furthermore, letters with transpositions are much easier to read than letter substitutions and this is critical for identifying what the word is or recognizing the word [8]. The main objectives 
of the current study were to investigate the effect of changing the level of positional noise and colors on reading performance for participants with BCVA equal to or better than 6/6 using non-recognizable words, called non-words and to examine how orthographic reading and word identification are affected by the use of non-words (pseudowords). We measured the reading speed, duration time for reading, and error percentage.

\section{Methodology}

In a cross-section interventional study, 20 English speakers (13 males and 7 females), aged 18-38 years with a mean age of 28.9 years, had bestcorrected distant visual acuity (BCVA) 6/6 or better were participated. Black print words were presented in a horizontal arrangement on cardboard with a white background (Figure 1). Two different stimuli were used. The text samples contained unrelated words of $3,4,5$, and 6 characters, presented in 9 lines using courier mono-spaced font and dark words on a white background. The distance between two adjacent words was two characters widths and the interline distance was five characters heights. In each trial, the pseudowords (non-words) were adapted and modified from the website "ARC Nonword Database" (http://www.maccs. mq.edu.au/ nwdb/nwdb.html) [9]. The viewing distance was $40 \mathrm{~cm}$. The words had an optimal font size of 12 pt (angular character size of $0.3^{\circ}$, defined as centerto-center spacing of horizontally adjacent characters) with contrast $>90 \%$. First, non-words were degraded using positional noise produced by random vertical displacements of the letter position below or above the horizontal line on three levels. Each vertical letter position was sampled from a Gaussian distribution with zero mean and variance in the range 0.00 (N0), 0.30 (N1), and 0.60 (N2) $\times$ character height ${ }^{2}$. Second, the wavelength was changed from short to long stimuli, including blue lighting (short), green lighting (mid), red lighting (long), and white lighting as everyday lighting, with constant illumination for all four different wavelengths (30 lux). The light levels were measured and controlled using an UPRtek spectrometer (MK350N Plus). The range of wavelengths measurable by the spectrometer (MK350N) is between 380 and $780 \mathrm{~nm}$. The participants were instructed to read the text samples aloud as fast as possible. They were given a brief demonstration of all the experimental conditions. Tests with non-words were presented in random order. The participants were video recorded while they were reading out loud. The total number of non-words read and the number of non-words reads incorrectly were counted. In the first experiment, the participants were asked to read three different texts with different levels of positional noise (N0, N1, and N2) under one level of lighting (30 lux) with non-words. The participants were

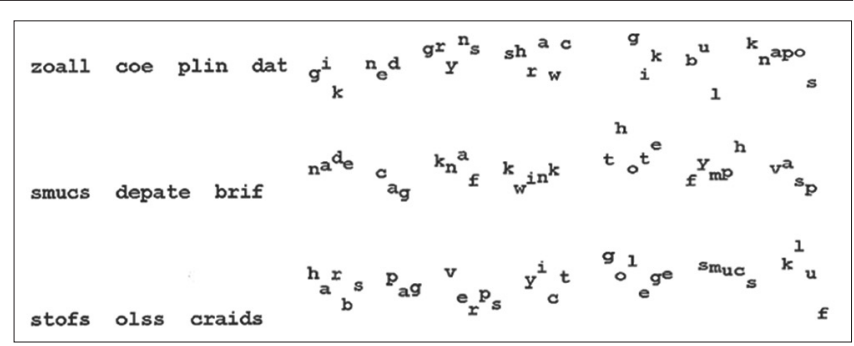

Figure 1: Sample of reading materials

asked to read each text for one minute. In the second experiment, the participants were asked to read under three different colored LED lighting conditions: Short (blue), mid (green), and long (red) wavelength. White LED light was also used (combining the same LED sources and fixed illumination of 30 lux). They were asked to read three different texts of non-words with N0, N1, and N2. The participants were asked to read each text for 1 min under each wavelength.

\section{Results}

We grouped the average reading rate for all the participants into real words and non-words to examine the effects of positional noise on reading performance. The average reading rate slowed down when the level of positional noise (N1 and N2) increased in both reading types (real and non-words). The statistical analysis showed that the reading rate varied between real words and non-words (one-way ANOVA, $\mathrm{P}=0.0003, \mathrm{~F}=15.15$, and $\mathrm{R}^{2}=0.39$ ). In real words, when the positional noise (N1) was introduced, the reading rate reduced, but the change was not statistically significant between $\mathrm{N} 0$ and $\mathrm{N} 1(\mathrm{P}=0.182)$. Furthermore, the reading rate reduced but the change was not statistically significant between $\mathrm{N} 1$ and $\mathrm{N} 2(\mathrm{P}=0.348)$. However, the reading rate reduced significantly between $\mathrm{N} 0$ and $\mathrm{N} 2$ (oneway ANOVA, $P=0.0004, F=15.15$ and $R^{2}=0.39$ ). In non-words, when the positional noise (N1) was introduced, the reading rate reduced but the change was not statistically significant between N0 and N1 $(P=0.44)$. Furthermore, the reading rate reduced, but the change was not statistically significant between N1 and $\mathrm{N} 2(\mathrm{P}=0.738)$. However, the reading rate reduced significantly between N0 and N2 (one-way ANOVA, $P=0.0199, F=15.15$, and $R^{2}=0.39$ ) (Figure 2).

The average word per minute rate was calculated for all the participants under the four different wavelengths and color. The reading rate did not vary much with the changes in wavelength, but the long-wavelength (red) was slower than the other wavelengths, but not significantly so (one-way ANOVA, $P=0.8746, F=0.23$, and $R^{2}=0.079$ ) (Figure 2-N0). For all four wavelengths, the average reading rate with non-words was slower than when reading real 
words and the statistical analysis showed that this was significant (one-way ANOVA, $\mathrm{P}=0.0011, \mathrm{~F}=9.6$, and $\mathrm{R}^{2}=0.31$ ). Furthermore, the results showed that when the wavelength changed from long (red) to mid (green) or short (blue), the mean difference reduced by an average of -9.6 words per minute. This change was statistically significant (one-way ANOVA, $P=0.0035$, $F=6.9$, and $R^{2}=0.19$ ). However, the mean difference was not significantly reduced between the mid and short wavelengths, as it reduced by an average of -1.2 words/min ( $p=0.905)$ (Figure 3).

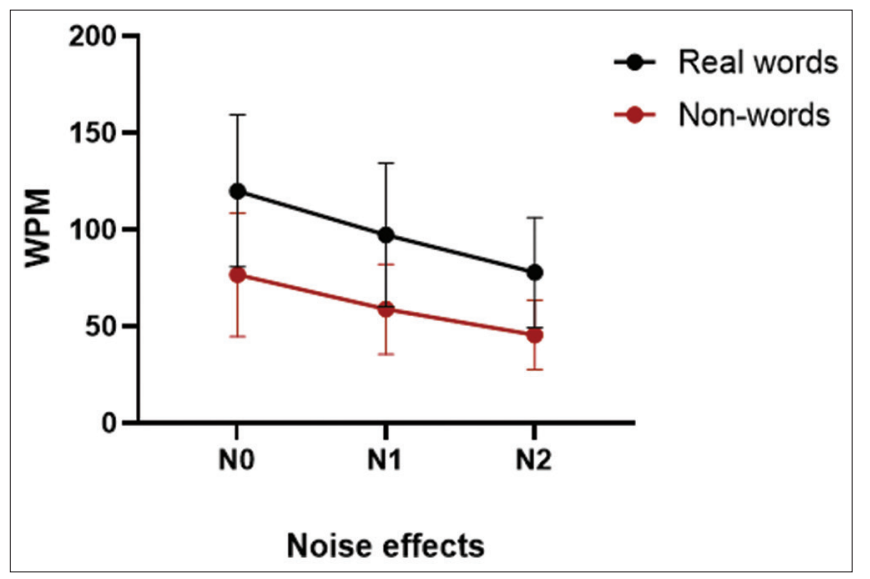

Figure 2: Mean reading rate $\pm S D$ under different levels of positional noise for real and non-words

When introducing positional noise, the average word per minute rate was calculated for all participants under four different colors and three different levels of positional noise. The statistical analysis showed that the reading rate was not significantly reduced in any wavelength when introducing positional noise between $\mathrm{NO}$ and $\mathrm{N} 1$ (one-way ANOVA, $\mathrm{P}=0.0358, \mathrm{~F}=7.9$, and $\mathrm{R}^{2}=0.28$ ) (Figure 3 ). However, the reading rate was significantly reduced in all wavelengths when positional noise was increased from N0 to N2 (oneway ANOVA, $p=0.0006, F=7.9$, and $R^{2}=0.28$ ) (Figure 4). The statistical analysis suggested that the mean difference in reading rate reduced when the wavelength changed from long to mid or short. This was significant, as it reduced by an average of -7.6 words/min (one-way ANOVA, $p=0.0016, F=8.8$, and $\left.R^{2}=0.24\right)$. However, there was no significant change

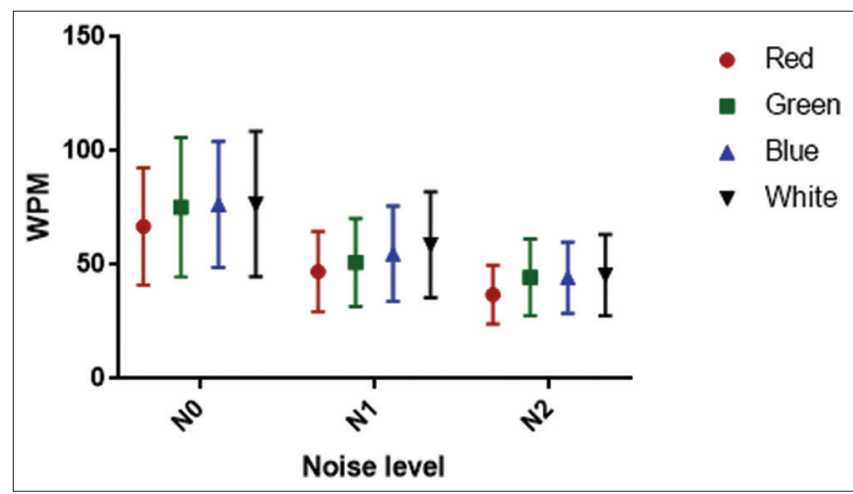

Figure 3: Mean reading rate $\pm S D$ under different wavelengths and noise effects in the mean difference between the mid and short wavelength with positional noise, as it reduced by an average of 0.15 words/min $(P=0.997)$ (Figure 5).

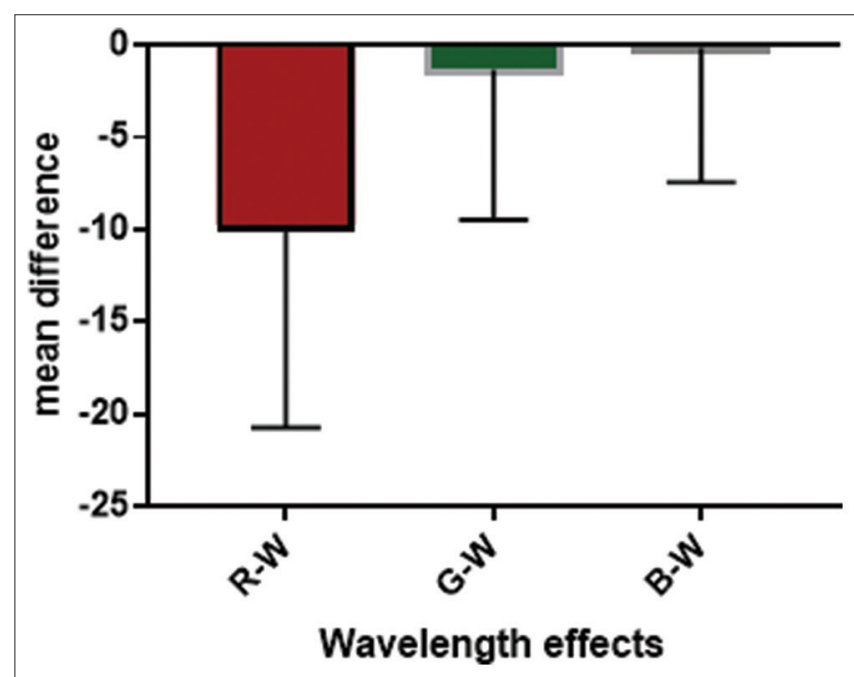

Figure 4: Mean differences in reading rate $\pm S D$ with in different wavelengths with NO

The linear regression tests were conducted on each color with and without positional noise. The reading rate as a function of positional noise was fitted with a linear function.

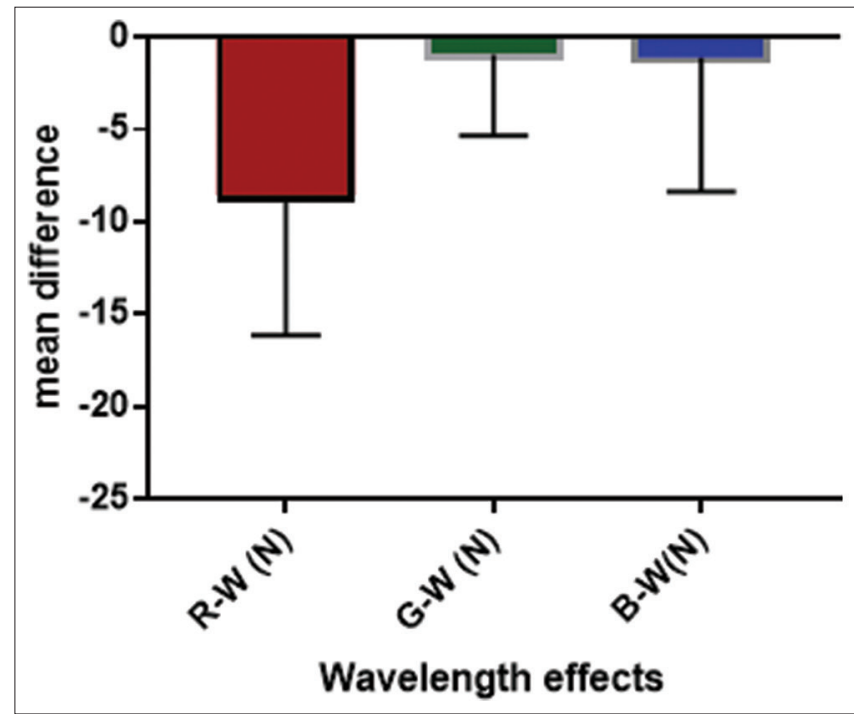

Figure 5: Mean differences in reading rate $\pm S D$ with different wavelengths with noise

Moreover, the reading performance reduced when positional noise was introduced in all wavelengths (Figure 6). Furthermore, the reading rate gradient and the initial reading rate for all colors were almost the same (parallel performance), except that the initial reading rate for the long-wavelength was slower than for the other wavelengths. The statistical analysis shows that there was no significant reduction in reading rate with or without positional noise in all wavelengths $\left(P=0.117, F=28.7\right.$, and $R^{2}=0.99$, which indicates $95 \%$ confidence interval) (Figure 6 ).

We analyzed the reading rate as a linear function of positional noise using the following equations: 

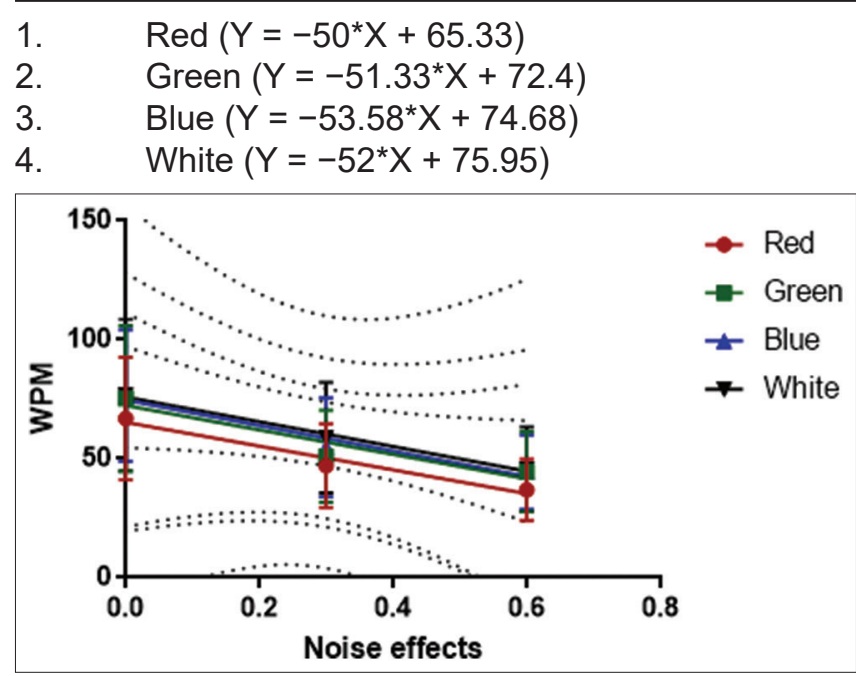

Figure 6: Linear regression function of reading rate with positional noise under different wavelengths

The error rate was similar across all conditions of different wavelengths for the same level of noise and statistically the changes were not significant $(P>0.999)$. However, the error rate increased when positional noise N1 and N2 were introduced, although there was no statistically significant difference between N0 and $\mathrm{N} 1(\mathrm{P}=0.625)$. With the long-wavelength, the error rate reduced significantly (one-way ANOVA, $P<0.0001$, $F=6.9$ and $R^{2}=0.28$ ) (Figure 7 ). The duration of word fixation was unchanged across the light levels for the same level of noise. In addition, the duration time was increased when introducing positional noise N1 and the statistical analysis showed that the reading rate was not significantly reduced in any of the wavelengths when positional noise between N0 and N1 was introduced $(p=0.185)$. However, the average duration time increased when positional noise N2 was introduced in all wavelengths and there was a statistically significant difference between N0 and N2 (Figure 8) (one-way ANOVA, $P<0.0001, F=8.1$, and $\left.R^{2}=0.28\right)$.

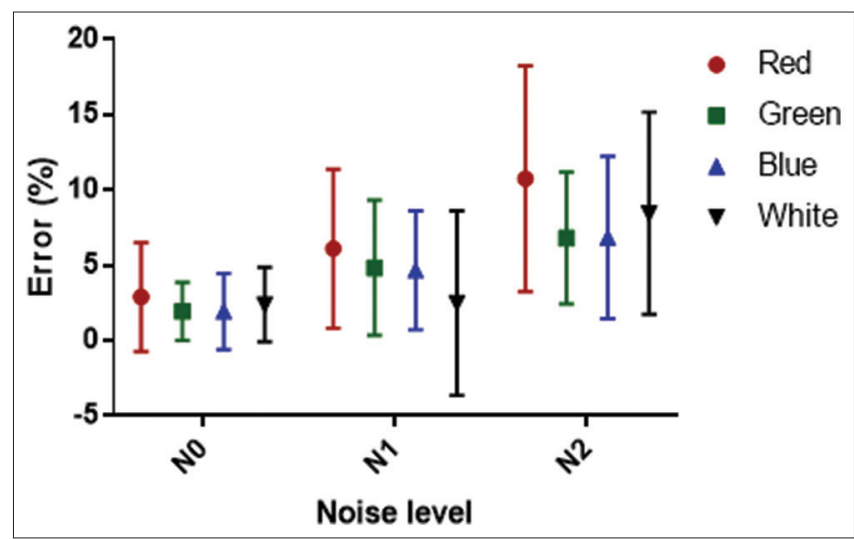

Figure 7: Mean reading error rate $\pm S D$ under different wavelengths and noise effects

\section{Discussion}

This study has shown that reading speed was significantly slower with non-words phonemically similar to real words than with real words and this finding was expected. This is due to the influence of the time that is needed for orthographic reading; the lack of some processes such as word recognition and reading comprehension can affect reading fluency. These results match those observed in earlier studies [6], [7], [10], [11], [12]. This finding could also be caused by the fact that cortical noise is created because of the lack of some processes (graphemeto-phoneme correspondence rules). Most spelling theories propose two major processes for translating between orthography and phonology: A lexical process for retrieving the spellings of familiar words (memorized words) and a sublexical (non-memorized word) process for assembling [1]. According to the "dual-route" models of reading, there are two separate mechanisms for processing words - lexical and sublexical. The lexical processing route, at the whole-word form level, provides a link between orthography (word template) and phonology decoding from lexical memory. It also enables the pronunciation of any regularly or irregularly spelled words, provided that these words have been previously memorized. The sublexical route conversion rules (traditionally grapheme-to-phoneme rules) perform the phonological decoding of printed letters into their spoken language by translating letters (graphemes) into their sounds (phonemes) [2]. This double dissociation between the lexical and sublexical mechanisms is the theoretical foundation of two functionally independent routes of reading at the wholeword level [1], [2], [3], [4], [5].

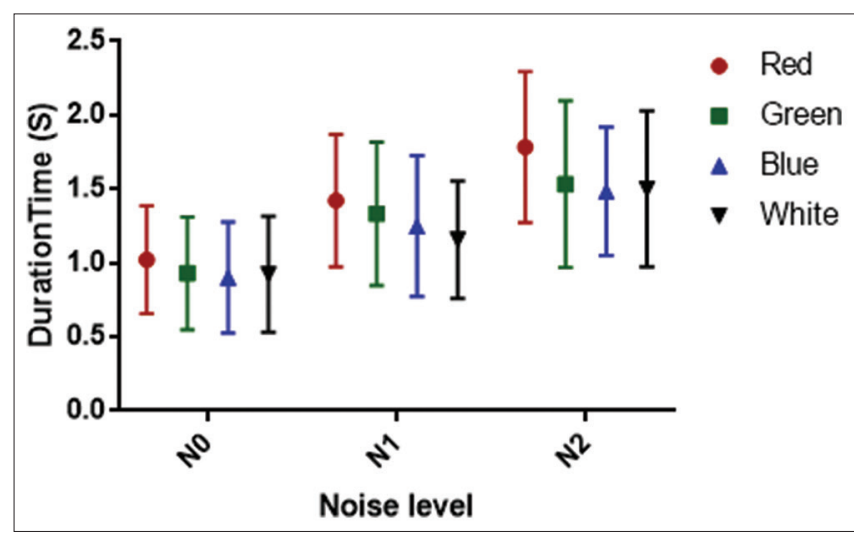

Figure 8: Mean duration 0 and noise effects

This study has shown that the reading speed of non-words was not significantly affected by different wavelengths of light for the participant readers. This is what we expected and consistent with the results of our previous study with changing colors [13]. Furthermore, the mean differences in wpm were reduced more with the long-wavelength compared to with the mid and short wavelengths, with and without positional noise. This is in line with the results of our previous study with real words [13], [14], [15], [16], [17]. This provided evidence for the fact that there is a cortical process that affects reading performance under long wavelengths. The findings of the current study for long-wavelength demonstrated that the reading performance under long-wavelength 
light (red light) was reduced or impaired, which is also consistent with [18], [19]. However, the reading rate for non-words under the short wavelength was no better than under the other wavelengths, as for real words, and that was different from what we expected. This is presumably because the noise level increases due to positional noise interfering with non-words [13], [14], [15], [16], [17], or it may be because reading parallel processing is different for lexical and sub-lexical items, and each has functionally independent routes of reading at the whole-word level [1], [2], [3], [4], [5]. This finding also provides evidence that different colors have different cortical effects by reducing the effects of positional noise on orthographic reading, word recognition, and word identification. When introducing positional noise, the reading rate decreased for all wavelengths, and this reduction did not vary with different colors. The findings of the current study were different from our previous study with real words and therefore, unexpected [13], especially for the short wavelength. This means that the effects of colors are varied as varied of cortical processing with different reading types and that was an interesting finding. This may be because of the increased noise levels or because positional noise interferes with non-words or disrupts word identification [13], [14], [15], [16], [17]. Furthermore, it may be because reading parallel processing is different for lexical and sub-lexical items, and each has functionally independent routes of reading at the whole-word level [1], [2], [3], [4], [5].

In this study, the results showed that the reading rates decreased with all wavelengths as a linear function for positional noise levels. This may be because of the increased noise levels or because positional noise interferes with non-words or disrupts word identification [13], [14], [15], [16], [17]. However, the long-wavelength reading rate function was the lowest linear function for positional noise levels compared with the other wavelengths. These findings further support the idea that reading performance under long-wavelength or red lighting is the lowest, regardless of reading types with and without positional noise. This is presumably due to the role of the magnocellular pathway function in letter recognition, which affects word recognition and word identification [19]. In addition, the reading rate gradient for shortwavelength lighting with non-words was different from that with real words, which was not what we expected. It may be that non-lexical words affect the ability of cortical processing to recognize the words or that the cortical reading process with non-lexical words is different from lexical word processing [3], [4], [5]. Furthermore, it is evidence that the enhancement in reading rate when reducing the effects of positional noise is not related to a small number of blue cones but to cortical processing. This study demonstrated that the detrimental effects of positional noise with non-words on reading rate were not influenced by changes in wavelengths and color. This was different from the effects of wavelengths with real words [13].
One possible explanation for this may be that reading parallel processing is different for lexical and sub-lexical items, and each has functionally independent routes of reading at the whole-word level; as a result, the findings were different according to the different types of cortical processing being used [1], [2], [3], [4], [5] Furthermore, this result may arise because the different reading processing starts in the late stage of cortical processing and this means that there is no role for the visual pathways, including the Magnocellular and Koniocellular pathways. Another possible explanation is that uncompleted word recognition (non-lexical) may create cortical noise or increased noise levels, or positional noise may interfere with non-words or disrupt word identification [13], [14], [20], [21]. Furthermore, the error rate was similar across all the different wavelengths when the level of positional noise was N0. When the level of noise was changed to N1 and N2, the error rate increased, but this increase did not reach statistical significance, except for the long-wavelength, which was obviously different from what we had expected. This is in line with our findings in a previous study with changing colors [13]. The increased error rate under long-wavelength light may occur because of the role of magnocellular processing in reading performance; it impairs the reading rate under red light, increasing the errors with unknown words [22].

The duration of word fixation was unchanged across the light levels with the same level of noise (N0). The duration of word fixation increased when the positional noise $\mathrm{N} 1$ was introduced, but this increased rate did not reach statistical significance. However, when introducing the positional noise N2, the duration of word fixation increased significantly. This is because orthographic reading takes longer to identify and recognize the word due to the effects of the positional noise [23].

\section{Acknowledgment}

We would like to thank the Optometry Department at Qassim University in Saudi Arabia for sponsorship of this study. Also, we would like to express our gratitude to the participants who have given their time willingly to participate in our research.

\section{Conclusion}

Reading performance is affected by changes in the levels of positional noise. However, the reading rate is not affected by changes in wavelength and color with non-words. The long-wavelength reading rate resulted in the lowest performance compared with other 
wavelengths and color with all levels of noise. The error rate and duration time increased when noise levels increased and there were different effects with different wavelengths even when positional noise was introduced.

\section{References}

1. Folk JR, Rapp B, Goldrick M. The interaction of lexical and sublexical information in spelling: What's the point? Cogn Neuropsychol. 2002;19(7):653-71. https://dl.acm.org/ doi/10.1145/3132525.3132546

PMid:20957558

2. Joubert SA, Lecours AR. The role of sublexical graphemic processing in reading. Brain Lang. 2000;72(1):1-13. $\quad$ https://doi.org/10.1006/brln.1999.2279 PMid:10716872

3. Sasisekaran J. Nonword repetition and nonword reading abilities in adults who do and do not stutter. J Fluen Disord 2013;38(3):275-89. https://doi.org/10.1016/j.jfludis.2013.06.001 PMid:24238389

4. Vigneau M, Jobard G, Mazoyer B, Tzourio-mazoyer N. Word and non-word reading: What role for the visual word form area? Neuroimage. 2005;27(3):694-705. https://doi.org/10.1016/j.neuroimage.2005.04.038 PMid:15961322

5. Ziegler JC, Besson M, Jacobs AM, Nazir TA, Carr TH. Word, pseudoword, and nonword processing: A multitask comparison using event-related brain potentials. J Cogn Neurosci. 1997;9(6):758-75. https://doi.org/10.1162/jocn.1997.9.6.758 PMid:23964598

6. BurtJS. What is orthographic processingskilland how does itrelate to word identification in reading? J Res Read. 2006;29(4):40017. https://doi.org/10.1111/j.1467-9817.2006.00315.x

7. Frost R, Katz L, Bentin S. Strategies for visual word recognition and orthographical depth: A multilingual comparison. J Exp Psychol Hum Percept Perform. 1987;13(1):104-15. PMid:2951484

8. Rayner K, White SJ, Johnson R, Liversedge S. Raeding wrods with jubmled lettres there is a cost. Psychol Res. 2006;17(3):1923. PMid:16507057 https://doi.org/10.1111/j.1467-9280.2006.01684.x

9. Rastle K, Harrington J, Coltheart M. 358,534 nonwords: The ARC nonword database. Q J Exp Psychol. 2002;55(4):133962. https://doi.org/10.1080/02724980244000099 PMid:12420998

10. Furr DL. In: House T, editor. Reading Clinic. Missouri: Truman House; 2000. p. 1-161.

11. Wegenhart TA. Better reading through science: Using researchbased models to help students read latin better. J Class Teach.
2015;16(31):8-13. https://doi.org/10.1017/s2058631015000021

12. Dehaene $S$, Cohen L, Sigman M, Vinckier F. The neural code for written words: A proposal. Trends Cogn Sci. 2005;9(7):335-41. https://doi.org/10.1016/j.tics.2005.05.004 PMid:15951224

13. Alsalhi A, Northway N, Walsh G, Elmadina AE. The effect of color and positional noise on reading performance in human vision. Open Access Maced J Med Sci. 2021;9(B):130-5. https:// doi.org/10.3889/oamjms.2021.5729

14. Pelli DG. Noise in the visual system may be early. In: Computational Models of Visual Processing. Cambridge: MIT Press; 1991. p. 147-52.

15. Gao X, Stine-Morrow EAL, Noh SR, Eskew RT. Visual noise disrupts conceptual integration in reading. Psychon Bull Rev. 2011;18(1):83-8. https://doi.org/10.3758/s13423-010-0014-4 PMid:21327368

16. Levi DM, Klein SA, Chen I. What limits performance in the amblyopicvisualsystem:Seeing signals in noisewithanamblyopic brain. J Vis. 2008;8(4):1-23. https://doi.org/10.1167/8.4.1 PMid:18484840

17. Rello L, Bigham JP. Good Background Colors for Readers: A Study of People with and without Dyslexia. ASSETS 2017 Proceedings $19^{\text {th }}$ International ACM SIGACCESS Conference on Computers and Accessibility; 2017. p. 72-80. https://doi. org/10.1145/3132525.3132546

18. Uccula A, Enna M, Mulatti C. Colors, colored overlays, and reading skills. Front Psychol. 2014;5:833. https://doi.org/10.3389/fpsyg.2014.00833 PMid:25120525

19. Chase C, Ashourzadeh A, Kelly C, Monfette S, Kinsey K. Can the magnocellular pathway read? Evidence from studies of color. Vision Res. 2003;43(10):1211$22 . \quad$ https://doi.org/10.1016/s0042-6989(03)00085-3 PMid:12705960

20. Cohen L, Dehaene S, Vinckier F, Jobert A, Montavont A Reading normal and degraded words: Contribution of the dorsal and ventral visual pathways. Neuroimage. 2008;40(1):35366. https://doi.org/10.1016/j.neuroimage.2007.11.036 PMid:18182174

21. Levi DM, Klein SA, Chen I. The response of the amblyopic visual system to noise. Vision Res. 2007;47(19):253142. https://doi.org/10.1016/j.visres.2007.06.014 PMid:17697689

22. Suttle C, Lawyenson J, Conway M. Efficacy of coloured overlays and lenses for treating reading difficulty: An overview of systematic reviews. Clin Exp Optom. 2018;101(4):514-20. https://doi.org/10.1111/cxo.12676 PMid:29633383

23. Pollatsek A, Reichle ED, Rayner K. Attention to one word at a time in reading is still a viable hypothesis: Rejoinder to Inhoff, Radach, and Eiter (2006). J Exp Psychol. 2006;32(6):1496500 https://doi.org/10.1037/0096-1523.32.6.1496 